\title{
An Analysis of Potential Predictors of Outcomes of Therapy for Urge Urinary Incontinence
}

Richter HE, Burgio KL, Chai TC, et al.: Predictors of outcomes in the treatment of urge urinary incontinence in women. Int Urogynecol J Pelvic Floor Dysfunct 2009, 20:489-497.

Rating: • Of importance.

Introduction: Overall success in the treatment of urge urinary incontinence may be predicated upon demographic, comorbid, and subjective factors that, in composite, could militate against or contribute to positive results after therapy administration. As part of a multi-institutional study, factors contributing to success after short-term therapy and the ability to discontinue therapy for urge urinary incontinence were assessed to determine whether certain pretreatment variables would be predictive of successful therapy.

Aims: To assess-as a planned review of data from a primary study-the factors that are predictive of shortterm success for urge urinary incontinence and whether therapy could be discontinued without resumption for a 6-month period.

Methods: This was a secondary analysis of data obtained from a prospective, multi-institutional, collaborative trial assessing 307 women who underwent primary therapy for urge urinary incontinence with pharmacologic treatment only or pharmacologic treatment combined with behavioral management techniques. Success was defined as at least $70 \%$ reduction of incontinence episodes at 10 weeks and the ability to then discontinue therapy and allow the patient to remain untreated for an additional 6 months.

Results: Multiple demographic (age, race, ethnic, income, occupation), medical historical, diary (number of incontinence episodes), symptomatic (Incontinence Impact Questionnaire scores, Overactive Bladder Questionnaire scores, Medical Epidemiologic and Social Aspects of Ageing scores), medical (drug use, chronic illness, diabetes), quality-of-life (12-item Short Form Health Survey), and examination (POP-Q [pelvic organ prolapse quantification system], pelvic floor muscle strength, and presence of fecal incontinence) factors were assessed. Only young age at treatment correlated with treatment success at 6 weeks. Six-month success was associated with only more significant anterior vaginal wall prolapse on the basis of POP-Q assessment.

Discussion: This study attempted to assess treatment success in a complex, sequential pattern using stringent outcomes criteria and alternating active and no-therapy time frames. The results were not conclusive as to which subgroups of urge urinary incontinence population might be better suited to receive monotherapy or combined therapy. The authors mentioned the possibility of creating more reasonable expectations of treatment as a possible positive outcome of this study.

\section{Comments}

The complex nature of the construct of this trial and the stringency with which success was defined resulted in very few meaningful factors being predictive of short-term or long-term treatment success. Additionally, the added requirement of retention of treatment benefit for an additional 6 months after cessation of active therapy further complicates interpretation of the dataset for predictive value. This study underscores the difficulty in studying the symptomatic urge urinary incontinence population. There almost certainly are unique subgroups within this population that we have not yet been able to delineate. Perhaps the issue is definition not based on response to therapy but rather on specific pretreatment syndrome factors associated with discreet comorbidities and psychosocial factors, such as willingness to persist with therapy and comply with behavioral change.

\section{Acknowledgment}

Dr. Roger R. Dmochowski is affiliated with the Department of Urology at Vanderbilt University Medical Center, where he is Director of Fellowship and the Section of Pelvic Reconstruction. Correspondence should be sent to A-1302 Medical Center North, Nashville, TN 37232, USA.E-mail: roger.dmochowski@vanderbilt.edu.

\section{Disclosure}

Dr. Dmochowski has served as a consultant for Astellas, Watson Pharmaceuticals, Pfizer, Novartis, Allergan, and Johnson \& Johnson. 